\title{
Seismic imaging and recognition technology of medium and shallow channel sand body
}

\author{
Dongna Wang* \\ PetroChina Jidong Oilfield company, Tangshan, Hebei 063004, China
}

\begin{abstract}
The fine characterization of channel sand body is a complex system engineering. The longitudinal superposition relationship of channel is complex, the sandstone thickness is thin, and the lateral changes of lithology and physical properties are obvious. It is necessary to carry out the research on the integration of processing and interpretation and seismic geology. The key technologies of seismic high-resolution pre stack and post stack inversion of medium and shallow channel sand bodies with "improved HHT point spectrum whitening" and "weak sidelobe wavelet structure" as the core and "azimuth well control inversion modeling" and "Cauchy regularization high-resolution inversion" as the core have been formed through experimental research.
\end{abstract}

key word: Channel sand; seismic high-resolution imaging processing; pre stack inversion; post stack inversion.

\section{Introduction}

The poor imaging, low resolution and low signal-to-noise ratio of existing seismic data in middle and shallow layers make it impossible to effectively resolve the thin-layer (5$10 \mathrm{~m}$ ) channel sand deposited in the river phase in middle and shallow layers, low coincidence rate predicted by reservoir inversion and paradoxically prominent well shock. Pilot investigations were conducted to form the key techniques for high-resolution imaging of meso shallow channel sand body earthquakes with cores of " improved HHT point spectrum albinism " and " weak paravalved daughter wave construction ", and for highresolution pre stack and post stack inversions with cores of " azimuthal well controlled Inversion Modeling " and ""Corsi regularized high-resolution inversion "[1].

\section{Key technology}

\subsection{Improved HHT point spectrum whitening technology Key technologies}

HHT point spectrum whitening technology is the key technology of post stack high-resolution processing. It includes three parts: time spectrum effective information extraction technology, high-frequency information optimization technology and fractal amplitude preserving technology[2]. The basic idea of HHT point spectrum whitening is shown in Figure 1.

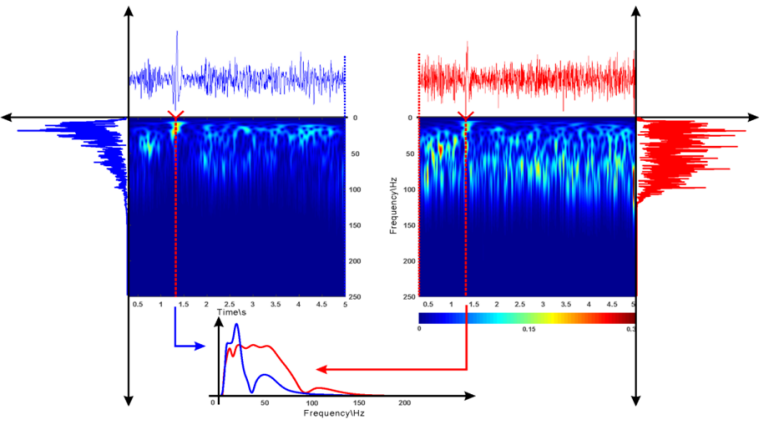

Fig. 1 Schematic diagram of HHT point spectrum whitening principle

(blue on the left - before high-resolution processing, red on the right - after high-resolution processing)

\subsection{Construction of weak sidelobe wavelet}

Seismic wavelet refers to the signal with stable waveform and length of about $60-100 \mathrm{~ms}$ formed after the pulse function excited by the source propagates for a period of time through geodetic filtering[3], resulting in wavelet distortion, especially the thin-layer tuning effect caused by wavelet sidelobe is the inevitable cause of error. In this study, a weak sidelobe mexh seismic wavelet (weak sidelobe Mexican Hat seismic wavelet) is constructed The construction method considers the influence of the sidelobe of the seismic wavelet on the seismic data processing link, considers the signal-to-noise ratio of the seismic section and the effective frequency band range to

\footnotetext{
* Corresponding author: wangdongna@petrochina.com.cn
} 
construct the wavelet, and designs the wavelet with the minimum sidelobe amplitude under the constraint of the effective frequency band width. It is of great value to improve the resolution and fidelity of seismic data. Through seismic high-resolution imaging processing, the resolution and amplitude retention of medium and shallow seismic data in matouying area are effectively improved. After high-resolution processing, the main frequency is increased from the original $15 \mathrm{~Hz}$ to $26 \mathrm{hz}$, the effective bandwidth[4] is expanded from the original 8$25 \mathrm{hz}$ to $5-45 \mathrm{hz}$, and the longitudinal resolution is increased from the original $44 \mathrm{~m}$ to $18 \mathrm{~m}$, which lays a foundation for the identification of thin channel sand bodies (as shown in Figure 2).

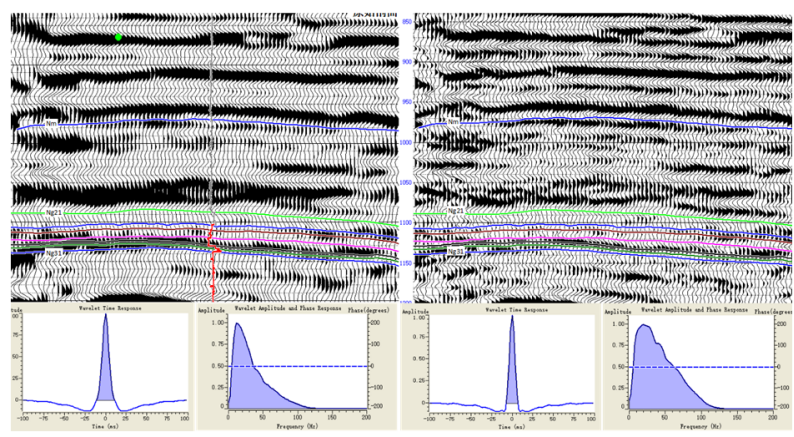

Fig. 2 cross section before (left) and after (right) high resolution processing of HHT point spectrum whitening (left - original profile, wavelet and spectrum, right - high resolution processing profile, weak sidelobe wavelet and spectrum)

\subsection{Azimuth well control Inversion Modeling Technology}

The logging data is broadband information in the vertical direction and has high resolution, which can accurately describe the detailed characteristics of the formation, but the horizontal distribution is relatively scattered [5]. It is necessary to match the constraints of each well to each seismic trace according to a certain weight. If geostatistical inverse distance weighted inversion modeling is applied, it will cause "bull's eye effect" (as shown in Figure 3), which is not conducive to the lateral fidelity of prestack seismic inversion. Azimuth constraints are introduced, and the shielding effect between wells is fully considered, so that the well can be extrapolated further and the modeling conforms to the sedimentary law.

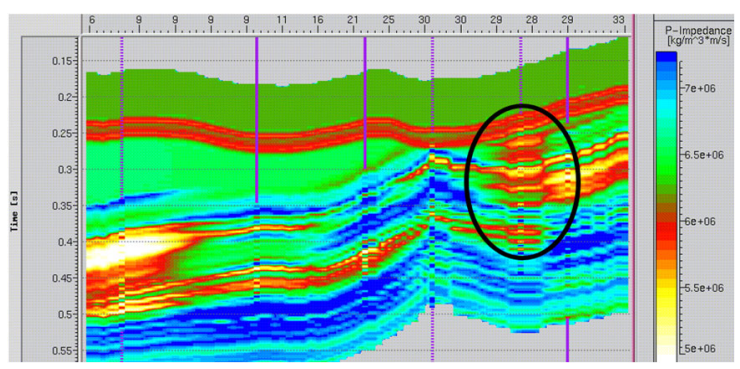

Fig. 3 Influence of bull eye effect on inversion

\subsection{Cauchy regularization high resolution inversion technique}

Compared with the existing Gaussian adaptive regularization, the Cauchy regularization high-resolution inversion technology proposed in this research has the following advantages: (1) the Gaussian distribution of the existing algorithm belongs to the "short tail" distribution, and the inversion results are mainly concentrated near the mean value, and the overall effect is relatively smooth; Cauchy distribution has the characteristics of "long tail" and constitutes a sparse constraint. Compared with Gaussian distribution, the vertical resolution of inversion is higher. (2) The block diagonal element of Gaussian regularization parameter is constant, and the block diagonal element of Cauchy regularization parameter changes adaptively[6]. Therefore, Cauchy regularization can produce different constraint coefficients for model parameters of different layers, further eliminate the statistical correlation between the three parameters, and improve the stability of prestack inversion.

\section{Application examples}

The high-resolution pre stack and post stack seismic inversion of medium and shallow channel sand body with high-precision logging modeling and constraint method[7], azimuth well control inversion, Cauchy regularization high-resolution inversion, shear wave prediction and multi angle joint inversion as the core has improved the prediction accuracy and resolution of medium and shallow channel sand body reservoir in Matou camp area, and the clear imaging of thin channel sandstone, The $3-5 \mathrm{M}$ thin channel sand reservoir is effectively identified [8]. By combining with the drilling data, the reservoir coincidence rate exceeds $90 \%$, which lays a foundation for the characterization of thin channel sand body (as shown in Fig. 4).

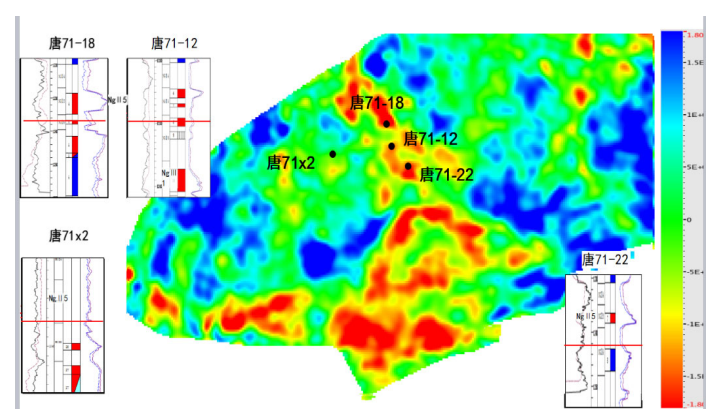

Fig. 4 distribution of medium and shallow channel and prediction of channel sand reservoir

The research results are applied to expand from the main structure to the structural wing, predict and depict the favorable river channel, and provide reliable support for the next drilling. As shown in Figure 5, the upper figure shows the river channel distribution and favorable river channel sand body prediction predicted by using the new processing data. Through the deployment and drilling of 
well T 71-39 and well T 71-35, thick oil layers were also drilled at the structural wing, which confirmed the amplitude preservation of the new data and the accuracy of river channel sand body prediction. The lower figure shows the data of traditional high-resolution processing, with great contradictions between well earthquakes and wells, It is difficult to identify the river distribution and boundary [9].

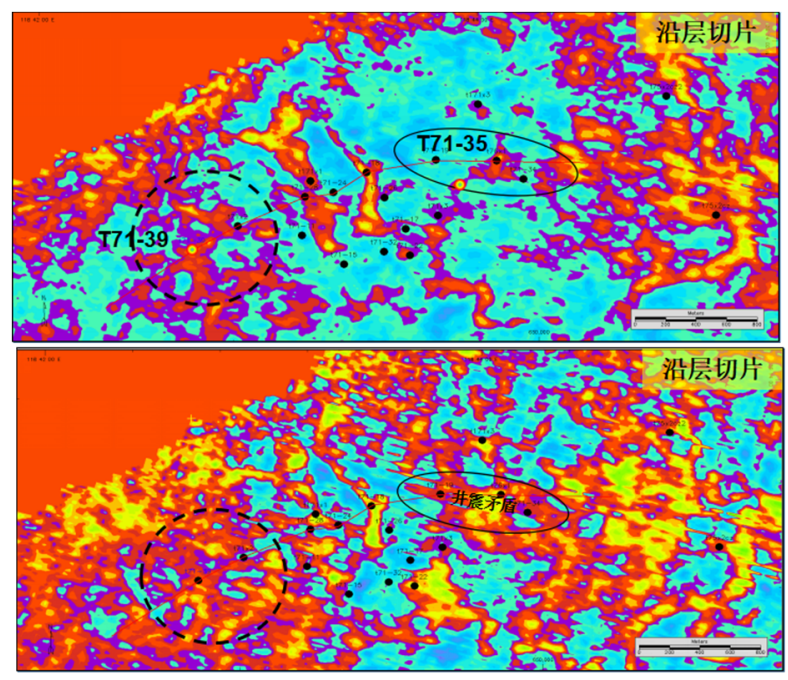

Fig. 5 channel distribution and channel sand reservoir prediction at the wing of matouying

(Upper: prediction chart of this key problem solving data, lower: prediction chart of previous key problem solving data)

\section{Conclusion}

This paper presents an integrated scheme of seismic data processing and interpretation based on geological targets. The seismic high-resolution imaging processing technology of medium and shallow channel sand body with "HHT point spectrum whitening high-resolution processing and weak sidelobe wavelet shaping" as the core has been formed, which improves the vertical resolution of medium and shallow seismic data and maintains the horizontal resolution, laying a seismic imaging foundation for the identification of thin interbedded channel sand body; Through the dynamic interpretation of sedimentary evolution based on standard layer, the dynamic evolution of channel sand body is recognized and characterized with high resolution.

Through the research and promotion of integrated processing and interpretation technology, it has effectively supported the construction of high-efficiency and high-quality production capacity in Nanpu Sag, greatly improved the exploration and development effect of mature exploration areas, and has reference significance for tapping the potential of similar seismic and geological conditions.

\section{References}

1. Zoeppritz K. Reflection and Propagation of Seismic Seismic Waves [J]. Gottinger Nachrichten, 1919(1). 66-84.

2. Stockwell RG, Mansinha L, Lowe R P. Localization of the complex spectrum : thestransform [J]. IEEE Transactions on Signal Processing, 1996, 44(4): 9981001.

3. Roberts A. Curvature attributes and their application to 3D interpreted horizons [J]. First Break, 2001, 19(2): 85-100.

4. CASTAGNA J P, SMITH S W. Comparison of AVO indicators: A modeling study [J]. Geophysics, 1993, 59(59): 1849-1855.

5. ALEMIE M W. Regularization of the AVO inverse problem by means of a multivariate Cauchy probability distribution [D], 2010.

6. DOWNTON J. Seismic parameter estimation from AVO inversion [M]. Ph D, University of Calgary, 2005.

7. Brossier R, Stéphane Operto, Virieux J . Velocity model building from seismic reflection data by fullwaveform inversion [J]. Geophysical Prospecting, 2014, 63(2): 354-367.

8. utton, S.P., and Loucks, R.D. 2010. Diagenetic controls on evolution of porosity and permeability in lower Tertiary Wilcox sandstones from shallow to ultradeep (200-6700 m) burial, Gulf of Mexico Basin, U.S.A.: Marine and Petroleum Geology, v.27, p.6981.

9. Bennion D B, Thoma F B, Imer D. (2000). Low Permeability Gas Reservoirs and Formation Damage -Tricks and Traps. SPE, 59753. DOI: 10.2118/59753MS. 